\title{
A SIMPLE METHOD OF HUMIDIFYING ANAESTHETIC GASES
}

\author{
J. E. WYNANDS, M.D., C.M., AND F. R. H. WRIGLEY, M.B., B.S., F.R.C.P.(c) ${ }^{\bullet}$
}

ANAESTHETTSTS HAVE RECOGNIZED for many years that the prolonged inspiration of dry anaesthetic gases may be one of the factors involved in the occurrence of postanaesthetic respiratory complications. A non-rebreathing technique may result in the loss of $28 \mathrm{mg}$. of water from the respiratory tract to every litre of inspired gas. ${ }^{1}$ In an effort to eliminate this factor, an effective method of humidifying inspired gases has been sought. By means of a Bennett Cascade Humidifier, it has been found possible to deliver to the patient anaesthetic gases fully saturated with water vapour at body temperature.

The Bennett Cascade Humidifier (Fig. 1) consists of a hot water reservoir through which the anaesthetic gases are bubbled. An adjustable thermostat is used to control two electric heating rods, which are inserted into casings which separate them from the water and the anaesthetic gases.

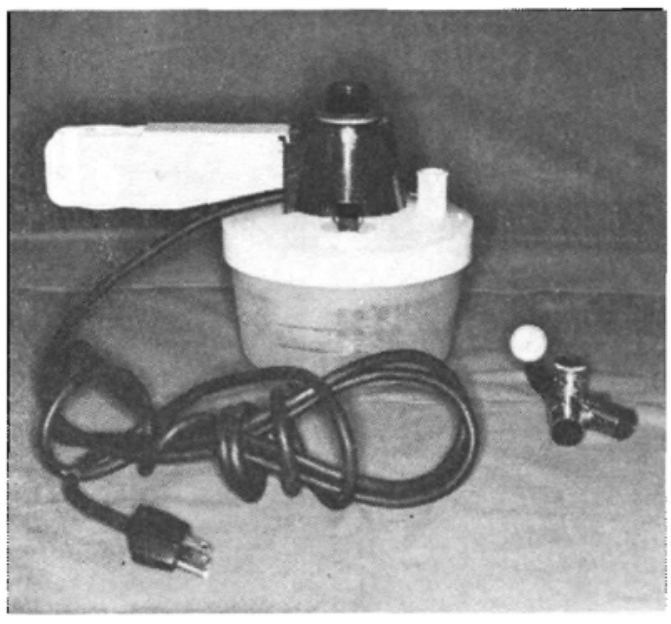

Figure 1

The humidifier can be connected in series with the inspiratory hose of a nonrebreathing system or it can be connected on the inspiratory side of a circle system (Fig. 2). High flows of fresh gas must be introduced into the circle in order that the carbon dioxide absorber may be excluded, for it has been found that soda lime becomes mushy when used in the circuit.

Fresh gases enter the humidifier, where they receive water vapour and are heated to well above body temperature; subsequently heat is lost in the hose connecting the humidifier to the endotracheal tube. A thermometer supplied

'Royal Victoria Hospital, Montreal 2. 


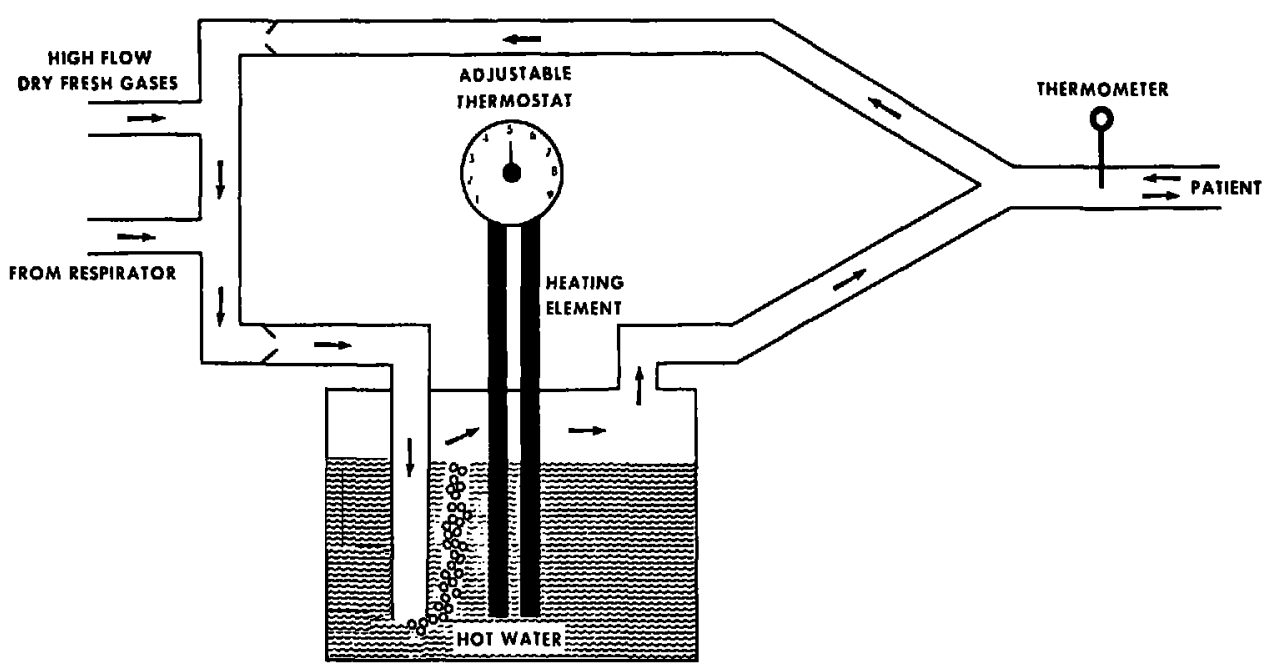

FiguRE 2

with the humidifier is inserted into the Y-connector of the circle to record the temperature of gases reaching the patient. By adjustment of the thermostat, gases can be delivered to the patient at body temperature, and since water precipitates from the gases as they cool, it can be assumed that they are saturated with water vapour on reaching the patient.

As water tends to collect in the inspiratory hose, it is important to avoid any situation which would allow the water precipitate to drain into the endotracheal tube. The corrugated tubes of the circle system should always be lower than the head of the patient, and the water collecting in them should be frequently drained. Figure 3 shows the humidifier attached to a bracket on the operating table. This appears to be the most satisfactory position for preventing the accumulation of water in the inspiratory tube.

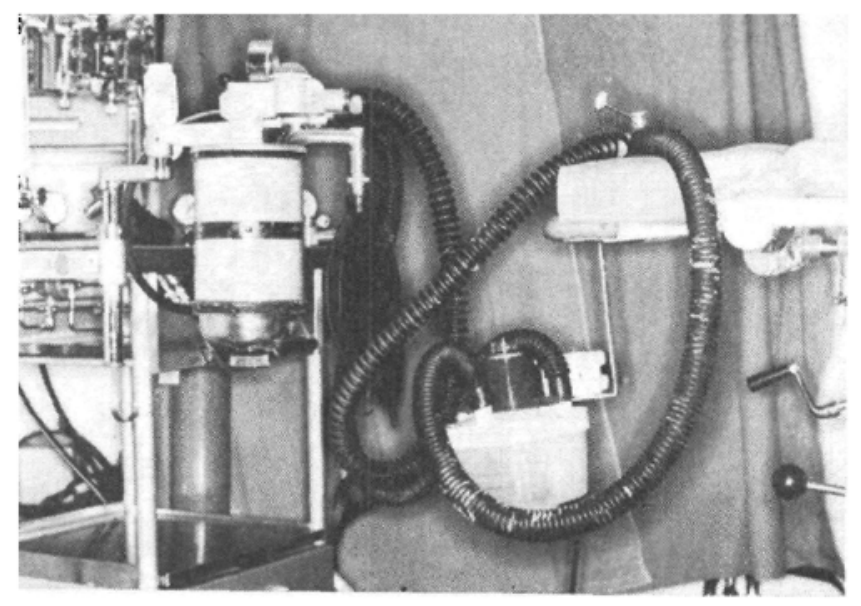

Figure 3 
The system is unsuitable for spontaneous ventilation techniques, since the anaesthetic gases are warmed and humidified by being passed through a volume of water. This causes an increase in inspiratory resistance equal to the depth of the water through which the gases pass, reaching a maximum of $5 \mathrm{~cm}$. of water. We have therefore chosen to employ this technique of humidification only when ventilation is being controlled.

The humidifier must be sterilized after each use, since the warm, humid atmosphere of the water reservoir provides an excellent culture medium for bacterial growth. Sterilization is easy: the heating element and thermostat are disconnected from the water reservoir, which is then dismantled. The component parts are then either autoclaved or soaked in 1 per cent Hibitaine diacetate solution for $\mathbf{2 0}$ minutes. They are then scrubbed in a detergent solution and thoroughly rinsed in water. There is no need to sterilize the heating element, as it does not come into contact with either the water or the anaesthetic gases. After sterilization, it is important to dry the components thoroughly, for pseudomonas and similar organisms may multiply in droplets left in the apparatus. For similar reasons, sterile distilled water should be used to fill the humidifier.

Since the apparatus contains an electrical heating element, it is considered unsuitable for use with flammable agents.

For accurate recordings of temperature the heat-sensitive portion of the thermometer probe should come into contact only with respired gases.

\section{REFERENCE}

1. Chase, H. F.; Kmmore, M. A.; \& Trotta, R. Respiratory Water Loss via Anaesthetic Systems: Mask Rebreathing. Anesthesiology. 22: 205 (1961). 